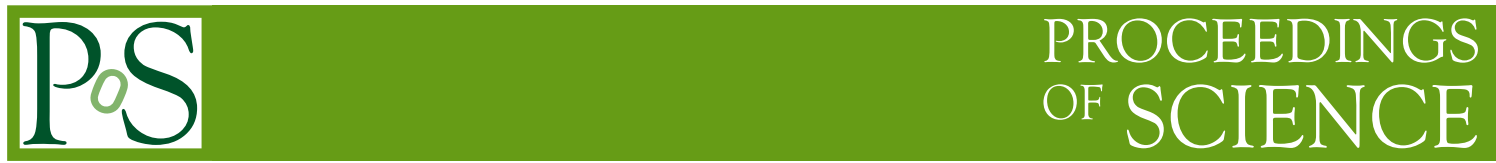

\title{
Black hole scaling relationships and NLS1s
}

\author{
Misty Bentz ${ }^{* \dagger}$ \\ Georgia State University \\ E-mail: bentz@chara.gsu.edu
}

\begin{abstract}
The vast majority of black hole masses that populate the literature are deduced from scaling relationships. Of these, the relationship between the radius of the broad line region and the luminosity of the AGN (the $r-L$ relationship) is perhaps the most widely used and is based on reverberation mapping experiments of low-redshift AGNs. Recent campaigns at MDM Observatory and Lick Observatory have revised and added several new reverberation measurements, some of which are based on observations of NLS1s. We describe preliminary results from ongoing work to recalibrate the $r-L$ relationship with these new reverberation results and high-resolution HST imaging. We also briefly describe ongoing work to recalibrate the scaling relationship between the black hole mass and host galaxy bulge luminosity for AGNs, the effects of host galaxy morphology on this relationship, and how the AGN relationship compares to that of the sample of quiescent galaxies with dynamical masses. We also investigate the narrow-line Seyfert 1s relative to the rest of the reverberation sample in the context of these scaling relationships.
\end{abstract}

Narrow-Line Seyfert 1 Galaxies and their place in the Universe

April 4-6, 2011

Milano, Italy

\footnotetext{
* Speaker.

${ }^{\dagger}$ With the MDM 2007 and LAMP 2008 teams.
} 


\section{Introduction}

Reverberation mapping (Blandford \& McKee 1982, Peterson 1993) is the most successful method for directly measuring the mass of the black hole in active galaxies. Through high-cadence spectrophotometric monitoring, the time delay between variations in the continuum flux and the echo of those variations in the broad emission line flux gives a measure of the size of the broad line region (BLR). Combined with the velocity width of the emission line itself, the black hole mass can be deduced, modulo a scaling factor that incorporates the details of the BLR such as the geometry and kinematics. Reverberation mapping has resulted in some 45 successful black hole mass measurements to date.

Recent reverberation campaigns have focused on two main issues: (1) replacing undersampled monitoring datasets to obtain more accurate BLR radius measurements, and (2) extending the range of BLR radii and black hole masses probed by the technique. In particular, the 2007 campaign at MDM Observatory (Denney et al. 2009, 2010) focused primarily on the former, and the 2008 Lick AGN Monitoring Project (LAMP; Bentz et al. 2008, 2009b) focused on the latter. The high quality BLR radius measurements from these two campaigns, combined with high-resolution Hubble Space Telescope images of the AGN host galaxies allows us to revisit several AGN scaling relationships and to compare the narrow-line Seyfert 1s (NLS1s) to the broad-line Seyfert 1s (BLS1s) in the reverberation database.

\section{Narrow-Line Seyfert $1 \mathrm{~s}$ in the Reverberation Database}

In their 1985 work, Osterbrock \& Pogge described a subsample of Seyfert 1 galaxies with broad Balmer components that were unusually narrow (FWHM $\lesssim 2000 \mathrm{~km} \mathrm{~s}^{-1}$ ). Two popular explanations for the NLS1 phenomenon are that the black holes are undermassive but currently accreting at a very high rate, or the inclinations of these AGNs are such that we are viewing them almost face-on. There does not yet seem to be a clear consensus as to which of these two pictures is favored by observations of NLS1s.

For this work, we classify an AGN as a NLS1 if FWHM $<2000 \mathrm{~km} \mathrm{~s}^{-1}$ for the broad $\mathrm{H} \beta$ com-

Table 1: NLS1s in the Reverberation Sample

\begin{tabular}{|c|c|c|c|c|c|c|}
\hline Object & $\begin{array}{c}\alpha_{2000} \\
\text { (hr min sec) }\end{array}$ & $\begin{array}{l}\delta_{2000} \\
\left(0^{\prime \prime \prime \prime}\right)\end{array}$ & $z$ & $\begin{array}{c}\left.\text { FWHM(H } \beta_{\text {broad }}\right) \\
\left(\mathrm{km} \mathrm{s}^{-1}\right)\end{array}$ & $\begin{array}{c}M_{\mathrm{BH}} \\
\left(10^{6} M_{\odot}\right)\end{array}$ & Ref. \\
\hline Mrk 335 & 000619.5 & +201210 & 0.02579 & $1792 \pm 3,1679 \pm 2$ & $14.2_{-3.7}^{+3.7}$ & 1 \\
\hline Mrk 110 & 092512.9 & +521711 & 0.03529 & $1543 \pm 5,1658 \pm 3,1600 \pm 39$ & $25.1_{-6.1}^{+6.1}$ & 1 \\
\hline NGC 4051 & 120309.6 & +443153 & 0.00234 & $799 \pm 2$ & $1.73_{-0.52}^{+0.15}$ & 2 \\
\hline PG $1211+143$ & 121417.7 & +140313 & 0.08090 & $2012 \pm 37$ & $: 146_{-44}^{+44}$ & 1 \\
\hline Mrk 202 & 121755.0 & +583935 & 0.02102 & $1471 \pm 18$ & $1.42_{-0.89}^{+0.85}$ & 3 \\
\hline Mrk 766 & 121826.5 & +294846 & 0.01293 & $1609 \pm 39$ & $1.8_{-1.4}^{+1.6}$ & 3 \\
\hline NGC 4748 & 125212.4 & -132453 & 0.01463 & $1947 \pm 66$ & $2.6_{-1.2}^{+1.0}$ & 3 \\
\hline NGC 7469 & 230315.6 & +085226 & 0.01632 & $1722 \pm 30$ & $12.2_{-1.4}^{+1.4}$ & 1 \\
\hline
\end{tabular}

References: 1. Peterson et al. (2004), 2. Denney et al. (2009), 3. Bentz et al. (2009b) 
ponent measured from the mean of all the spectra obtained during a reverberation campaign. ${ }^{1}$ The eight AGNs that satisfy this criterion are listed in Table 1. Several are famous NLS1s which have been the objects of intense scrutiny, such as NGC 4051 and Mrk 766. However NGC 7469, which is usually classified as a BLS1, was apparently in a NLS1-type phase during its 1996 monitoring campaign and is therefore included as a NLS1 here.

\section{The Radius - Luminosity Relationship}

The relationship between the radius of the broad line region and the luminosity of the AGN (the $r-L$ relationship; Kaspi et al. 2000, Bentz et al. 2009) is the most utilized scaling relationship to result from the compilation of reverberation measurements. It allows one to quickly estimate the mass of a black hole with two simple measurements from a single spectrum: the continuum luminosity as a proxy for the observationally-intensive measurement of the BLR radius, and the velocity width of a broad emission line.

AGN monitoring apertures are typically quite large for reverberation mapping campaigns (e.g., $5^{\prime \prime} \times 8^{\prime \prime}$ ) to minimize the effects of variable seeing and slit losses. However, since many of the reverberation-mapped AGNs are hosted by nearby, bright galaxies, a large monitoring aperture also collects a large amount of host-galaxy starlight. This has the effect of artificially steepening the slope of the $r-L$ relationship, as the starlight dilution is worst for the nearest AGNs which tend to be relatively low-luminosity and have small BLR radii. The starlight component of the spectral luminosity can be determined from two-dimensional surface brightness modeling of high-

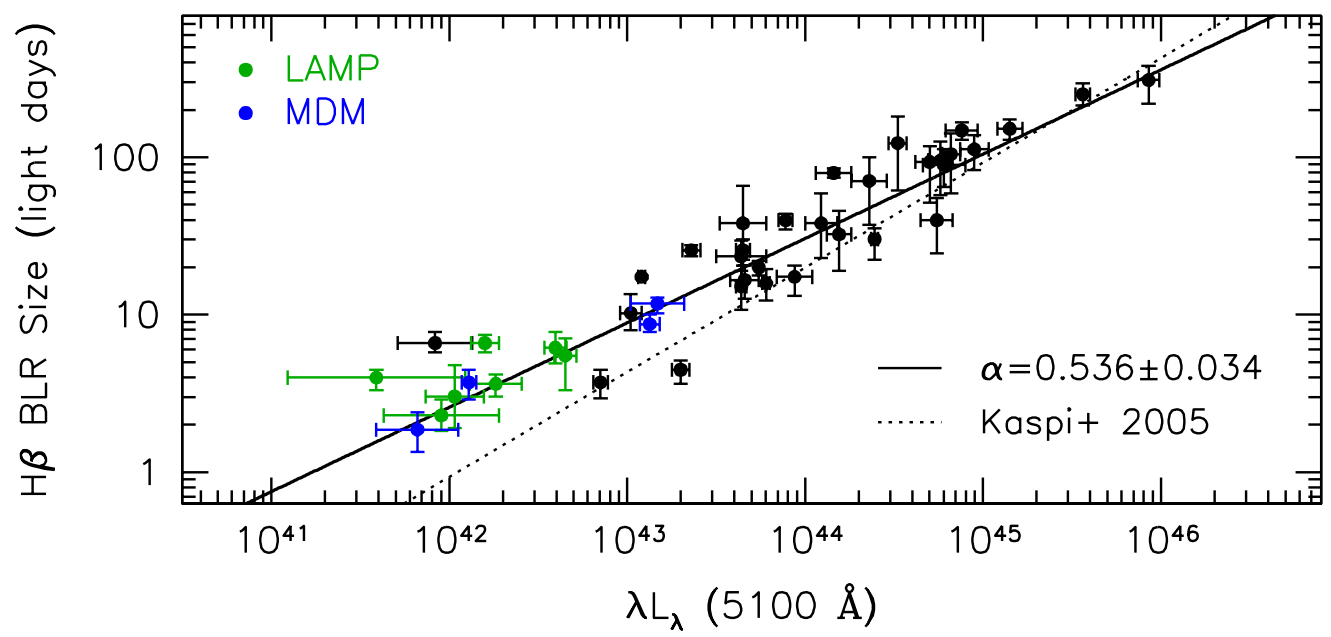

Figure 1: The $\mathrm{H} \beta r-L$ relationship for AGNs after subtracting the contribution from host-galaxy starlight. The AGNs in the LAMP sample are shown in green and their luminosities have been corrected using preliminary fits to HST WFC3 images. The AGNs from the MDM sample are shown in blue. The best fit is shown by the solid line and has a powerlaw slope of $0.536 \pm 0.034$, consistent with the results of Bentz et al. (2006, 2009). For comparison, we also plot the best fit relationship from Kaspi et al. (2005; dotted line) which does not include any correction for host-galaxy starlight.

\footnotetext{
${ }^{1}$ For the Peterson et al. (2004) compilation, linewidths measured from the mean spectra are tabulated by Collin et al. (2006).
} 


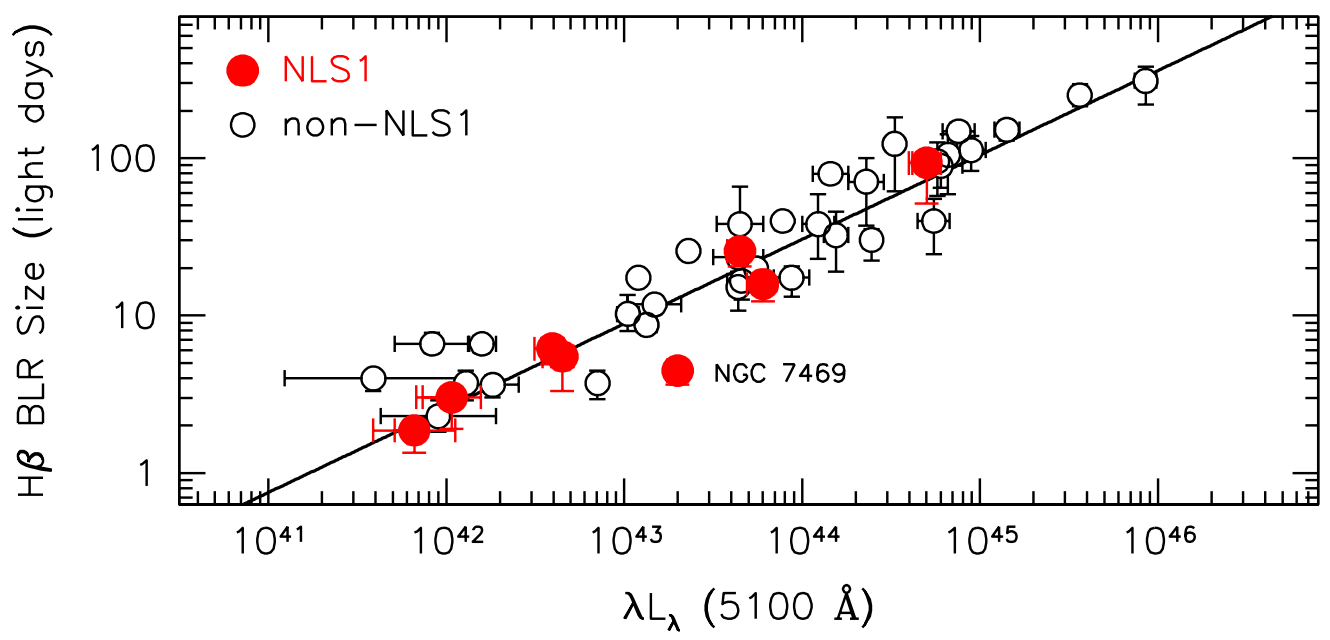

Figure 2: Same as Figure 1, except the red circles show the NLS1s in the reverberation sample. There is no obvious difference between the locations of the NLS1s and the BLS1s in the reverberation sample.

resolution Hubble Space Telescope images (Bentz et al. 2006, 2009). When the luminosities are corrected for this component, the slope of the $r-L$ relationship is found to be $\alpha \approx 0.5$, consistent with the value expected from simple photoionization arguments.

In Figure 1 we display the most recent determination of the $\mathrm{H} \beta r-L$ relationship for all reverberation-mapped AGNs with HST imaging. Surface brightness modeling of new HST WFC3 images is currently being carried out for the objects from the LAMP 2008 campaign and one new object from the MDM 2007 campaign (Bentz et al. 2011, in preparation), but we can make preliminary corrections to their luminosities. The best-fit powerlaw slope of $0.536 \pm 0.034$ is consistent with the slopes reported by Bentz et al. (2006, 2009). Even with the addition of several objects at the low luminosity end, the relationship shows no signature of a turnover at small radii or low luminosities.

Figure 2 shows the $r-L$ relationship with the locations of the NLS1s highlighted relative to the BLS1s. The NLS1s are distributed evenly about the best fit to the $r-L$ relationship and have a similar scatter to the BLS1s in the sample. There appears to be no difference between the NLS1s and BLS1s, which implies that the physics of the BLR is similar for NLS1s and BLS1s. The sometimes-NLS1 galaxy NGC 7469 is currently the most discrepant object in the $r-L$ relationship, however a new monitoring campaign has recently been completed for this AGN in the hopes of obtaining higher quality spectrophotometry (Grier et al., in preparation), so this object's apparent discrepancy is still being investigated. We emphasize that the good agreement between the $r-L$ relationship for BLS1s and NLS1s is only known to be true for the optical-emitting region of the BLR, as we do not currently have enough information to carry out the same study for the UVemitting BLR.

\section{The Black Hole Mass - (Pseudo)Bulge Luminosity Relationship}

Scaling relationships between the black hole and properties of the host galaxy are also widely used but many of these were originally determined from quiescent galaxies with dynamical black 
hole mass measurements (i.e., Magorrian et al. 1998, Ferrarese at al. 2000). Recently, there have been several studies claiming that host-galaxy morphology may affect the details of these scaling relationships, and in particular, the presence of a pseudobulge or a bar. Both pseudobulges and bars are interpreted as signatures of host-galaxy secular evolution, in which disk instabilities drive gas to the center rather than the build-up of a classical bulge through mergers. Recently, Kormendy et al. (2011) reported that there is no correlation between black hole mass and pseudobulge luminosity or pseudobulge velocity dispersion. In direct conflict, however, are the findings of $\mathrm{Hu}$ (2008), who show that pseudobulges in the same sample do show an $M_{\mathrm{BH}}-\sigma_{*}$ relationship, but that it lies below the relationship for ellipticals and classical bulges. Graham et al. (2011) report a similar offset for the $M_{\mathrm{BH}}-\sigma_{*}$ relationship for barred galaxies in the same sample when compared to the $M_{\mathrm{BH}}-\sigma_{*}$ relationship for unbarred galaxies, and go on to speculate that if there is no morphology-based offset found in the relationship between black hole mass and bulge luminosity, then that implies that there may be a problem with using velocity dispersion measurements to estimate black hole masses.

Surface brightness modeling of high-resolution optical HST images for the reverberation sample allows us to investigate the $M_{\mathrm{BH}}-L_{\text {bulge }}$ relationship for active galaxies, which we show in Figure 3. We plot the $V$-band luminosities of classical bulges and ellipticals versus those of pseudobulges (Figure 3 left) as well as for unbarred galaxies versus barred galaxies (Figure 3 right). The pseudobulges and the barred galaxies seem to follow the same $M_{\mathrm{BH}}-L_{\text {bulge }}$ relationship as the classical bulges and the unbarred galaxies with no apparent offsets, and also seem to follow the same relationship as has been found for the non-spiral hosts of quiescent galaxies with dynamical black hole mass measurements (solid line, Gültekin et al. 2009). This would seem to imply that the $M_{\mathrm{BH}}-L_{\text {bulge }}$ relationship is relatively insensitive to the details of host galaxy morphology and may actually be a more accurate way to estimate the black hole mass from an observable host galaxy characteristic. The pseudobulges do, however, show increased scatter about the relationship, which is perhaps not surprising given the fact that we have measured the luminosities from optical images and pseudobulges often have large amounts of nuclear dust and ongoing star formation. The larger scatter and small dynamic range of the pseudobulges in the quiescent galaxy sample (Figure 3 left, gray points) is likely the reason Kormendy et al. (2011) fail to find a relationship between black hole mass and pseudobulge luminosity.

We also investigate the location of the NLS1s in the $M_{\mathrm{BH}}-L_{\text {bulge }}$ relationship. Three of the eight NLS1s are hosted by classical bulges, while the other five reside in pseudobulges. The NLS1s do not show an offset from the BLS1s and seem to be evenly distributed within the scatter exhibited by the BLS1s. Again, the most discrepant object is NGC 7469, which we have discussed above. The good agreement between the NLS1s and BLS1s in the sample would seem to imply that NLS1s, or at least those currently included in the reverberation sample, are not undermassive relative to their host galaxies and are as often found in pseudobulges as they are found in classical bulges. However, it is important to keep in mind that these preliminary results are based on optical images. We intend to obtain high-resolution near-IR imaging of the entire reverberation sample to further this investigation. 

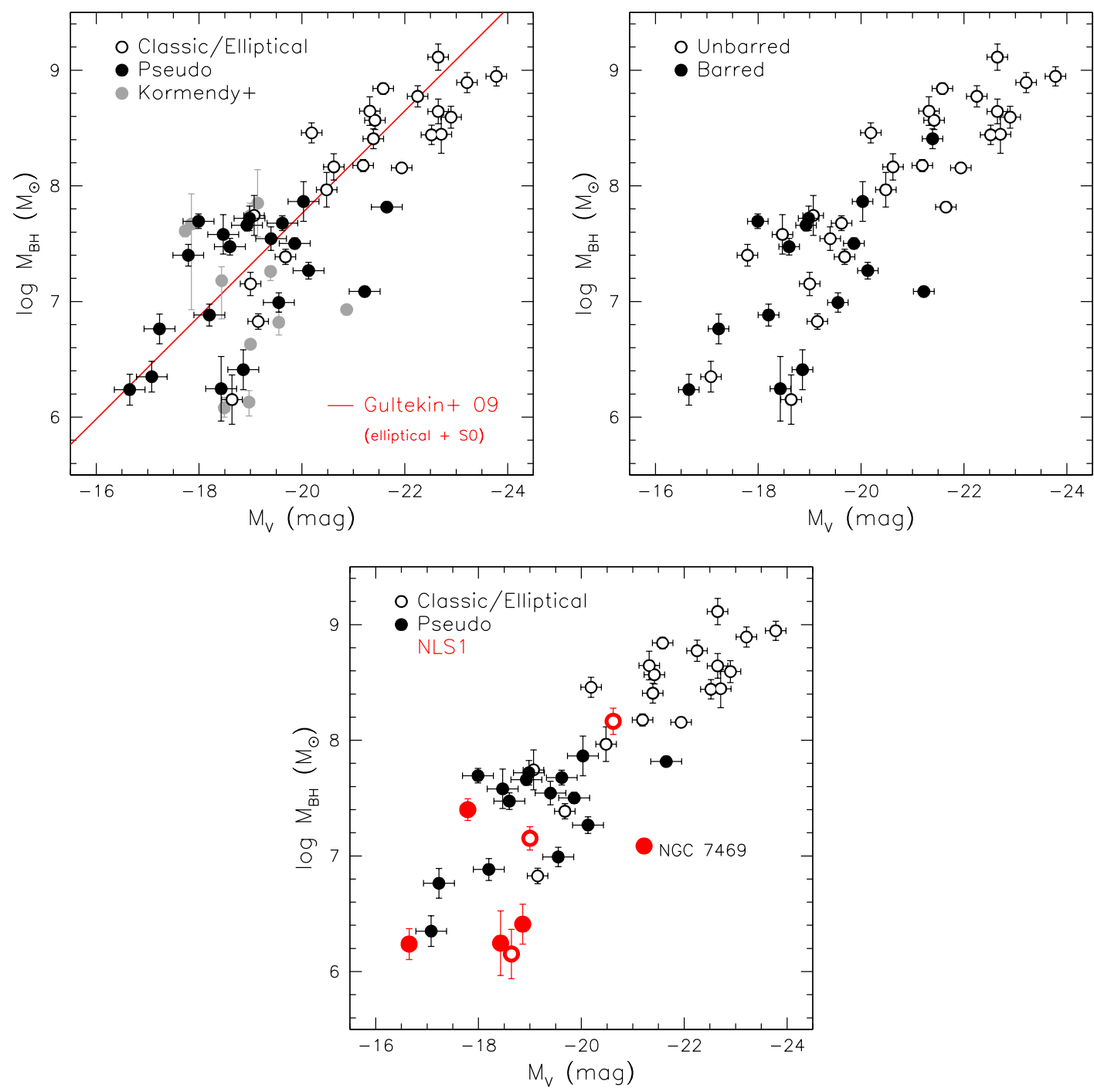

Figure 3: Black hole mass - bulge luminosity relationship for the AGNs in the reverberation sample based on two-dimensional surface brightness fits to high-resolution, optical HST images. In all three plots, the scales and the axes are the same. Left: Elliptical galaxies and galaxies with classical bulges are displayed with open circles, galaxies with pseudobulges are shown with filled circles, and the pseudobulge galaxies from the quiescent galaxy sample of Kormendy et al. (2011) are shown in gray for comparison. The solid red line is the best fit from Gültekin et al. (2009) to the non-spiral quiescent galaxies (not shown). The active galaxies appear to follow the same relationship as the quiescent galaxies regardless of morphological type. There does appear to be a modest increase in scatter among the AGN pseudobulge galaxies that may be partially due to dust extinction and bright star forming regions, both of which can affect the luminosity measured from optical images. Right: AGN host galaxies without bars are shown with open circles while barred galaxies are shown with filled circles. Again, there appears to be no offset based on galaxy morphology. Bottom: NLS1s are shown in red, with open circles denoting a classical bulge or elliptical host galaxy, and filled circles denote a host galaxy with a pseudobulge. The NLS1s seem to follow the same relationship as the BLS1s and exhibit a similar scatter. 


\section{Summary}

Recent reverberation mapping campaigns have reduced the scatter and extended the range at the low end of black hole scaling relationships in active galaxies. A preliminary update to the optical $r-L$ relationship finds a slope that is consistent with $\alpha \approx 0.5$ and with slopes previously determined where the host galaxy starlight contribution is accounted for. The optical $M_{\mathrm{BH}}-L_{\text {bulge }}$ relationship appears to be relatively insensitive to the host-galaxy morphology, in contrast to recent studies that find morphology-based offsets in the $M_{\mathrm{BH}}-\sigma_{*}$ relationship for quiescent galaxies with dynamical masses. Finally, NLS1s in the reverberation sample appear to follow the same scaling relationships as BLS1s and exhibit a similar scatter about the best-fit relationships.

\section{References}

[1] M.C. Bentz, B.M. Peterson, R.W. Pogge, M. Vestergaard, \& C.A. Onken, The Radius-Luminosity Relationship for Active Galactic Nuclei: The Effect of Host-Galaxy Starlight on Luminosity Measurements, 2006, ApJ, 644, 133.

[2] M.C. Bentz, et al., First Results from the Lick AGN Monitoring Project: The Mass of the Black Hole in Arp 151, 2008, ApJ, 689, L21.

[3] M.C. Bentz, B.M. Peterson, H. Netzer, R.W. Pogge, \& M. Vestergaard, The Radius-Luminosity Relationship for Active Galactic Nuclei: The Effect of Host-Galaxy Starlight on Luminosity Measurements. II. The Full Sample of Reverberation- Mapped AGNs, 2009a, ApJ, 697, 160.

[4] M.C. Bentz, et al., The Lick AGN Monitoring Project: Broad-line Region Radii and Black Hole Masses from Reverberation Mapping of $H \beta, 2009 \mathrm{~b}, \mathrm{ApJ}, 705,199$.

[5] R.D. Blandford \& C.F. McKee, Reverberation mapping of the emission line regions of Seyfert galaxies and quasars, 1982, ApJ, 255, 419.

[6] S. Collin, T. Kawaguchi, B.M. Peterson, M. Vestergaard, Systematic effects in measurement of black hole masses by emission-line reverberation of active galactic nuclei: Eddington ratio and inclination, 2006, A\&A, 456, 75.

[7] K.D. Denney, et al., A Revised Broad-line Region Radius and Black Hole Mass for the Narrow-line Seyfert 1 NGC 4051, 2009, ApJ, 702, 1353.

[8] K.D. Denney, et al., Reverberation Mapping Measurements of Black Hole Masses in Six Local Seyfert Galaxies, 2010, ApJ, 721, 715.

[9] L. Ferrarese \& D. Merritt, A Fundamental Relation between Supermassive Black Holes and Their Host Galaxies, 2000, ApJ, 539, L9.

[10] A.W. Graham, C.A. Onken, E. Athanassoula, F. Combes, An expanded $M_{\mathrm{BH}}-\sigma$ diagram, and a new calibration of active galactic nuclei masses, 2011, MNRAS, 412, 2211.

[11] K. Gültekin, et al., The $M-\sigma$ and $M-L$ Relations in Galactic Bulges, and Determinations of Their Intrinsic Scatter, 2009, ApJ, 698, 198.

[12] J. Hu, The black hole mass-stellar velocity dispersion correlation: bulges versus pseudo-bulges, 2008, MNRAS, 386, 2242.

[13] S. Kaspi, P.S. Smith, H. Netzer, D. Maoz, B.T. Jannuzi, U. Giveon, Reverberation Measurements for 17 Quasars and the Size-Mass-Luminosity Relations in Active Galactic Nuclei, 2000, ApJ, 533, 631. 
[14] S. Kaspi, D. Maoz, H. Netzer, B.M. Peterson, M. Vestergaard, \& B.T. Jannuzi, The Relationship between Luminosity and Broad-Line Region Size in Active Galactic Nuclei, 2005, ApJ, 629, 61.

[15] J. Kormendy, R. Bender, M.E. Cornell, Supermassive black holes do not correlate with galaxy disks or pseudobulges, 2011, Nature, 469, 374.

[16] J. Magorrian, et al., The Demography of Massive Dark Objects in Galaxy Centers, 1998, AJ, 115, 2285.

[17] D.E. Osterbrock \& R.W. Pogge, The spectra of narrow-line Seyfert 1 galaxies, 1985, ApJ, 297, 166.

[18] B.M. Peterson, Reverberation mapping of active galactic nuclei, 1993, PASP, 105, 247.

[19] B.M. Peterson, Central Masses and Broad-Line Region Sizes of Active Galactic Nuclei. II. A Homogeneous Analysis of a Large Reverberation-Mapping Database, 2004, ApJ, 613, 682. 